\title{
Quantification of Biological Tissue and Construction of Patient Equivalent Phantom (Skull and Chest) for Infants (1-5 years old)
}

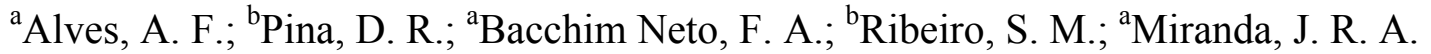 \\ ${ }^{a}$ Dept of Physics and Biophysics, Botucatu Biosciences Institute, Univ Estadual Paulista UNESP, \\ Distrito de Rubião Junior S/N, Botucatu, São Paulo, Brazil 18618-000. \\ ${ }^{\mathrm{b}}$ Dept of Tropical Diseases and Imaging Diagnoses, Botucatu Medical School, Univ Estadual \\ Paulista UNESP, Distrito de Rubião Junior S/N, Botucatu, São Paulo, Brazil 18618-000.
}

\begin{abstract}
Our main purpose in this study was to quantify biological tissue in computed tomography (CT) examinations with the aim of developing a skull and a chest patient equivalent phantom (PEP), both specific to infants, aged between 1 and 5 years old. This type of phantom is widely used in the development of optimization procedures for radiographic techniques, especially in computed radiography (CR) systems. In order to classify and quantify the biological tissue, we used a computational algorithm developed in Matlab ${ }^{\circledR}$. The algorithm performed a histogram of each CT slice followed by a Gaussian fitting of each tissue type. The algorithm determined the mean thickness for the biological tissues (bone, soft, fat, and lung) and also converted them into the corresponding thicknesses of the simulator material (aluminum, PMMA, and air). We retrospectively analyzed 148 CT examinations of infant patients, 56 for skull exams and 92 were for chest. The results provided sufficient data to construct a phantom to simulate the infant chest and skull in the posterior-anterior or anterior-posterior (PA/AP) view. Both patient equivalent phantoms developed in this study can be used to assess physical variables such as noise power spectrum (NPS) and signal to noise ratio (SNR) or perform dosimetric control specific to pediatric protocols.
\end{abstract}

Keywords: tissue quantification, computational algorithm, patient equivalent phantom, chest phantom, skull phantom, pediatric radiology, pediatric phantom.

\section{INTRODUCTION}

Pediatric patients between one and five years old are often submitted to radiological examinations. Skull and chest radiographies are the most frequently ones performed due to head trauma and pneumonia, respectively [1-2]. Infant patients have a higher risk of developing genetic alterations that may result in some type of cancer induced by radiation exposure due to their greater radiosensitivity tissues and increased life expectancy [3]. Therefore, the optimization of radiographic techniques for pediatric patients ensures the quality of radiographic examinations associated with low doses of radiation [4].

Homogeneous phantoms constitute an important tool in the optimization process of radiography systems. The patient equivalent phantom (PEP) is one of the most widely used instrument due to some of its characteristics. PEP is lightweight, portable, made of readily available materials, and also able to accurately simulate the standard patient's attenuation over a representative range of tube potentials [5-6]. The American Association of Physicist in Medicine (AAPM) Report no.31 (1990) [7] describes the materials and the construction of both ANSI chest and ANSI skull patient equivalent phantoms for standard patients. These devices are composed of polymethyl methacrylate (PMMA) and aluminum, which are easily available materials [7-8].

Pina et al (2012) proposed the construction of pediatric homogeneous phantoms based on the biological tissue quantification applied to retrospective computed tomography examinations of newborn patients (age $<1$ year) [9]. From this study, was developed a computational algorithm to classify and quantify biological tissues in the chest region. The algorithm performed the patient examination histogram analyses and, through the Gaussian fit, differentiates biological tissues (lung, soft, fat and bone tissues) and converts those tissues into simulator materials such as PMMA and aluminum [9].

In this work, we applied the methodology described by Pina et al (2012) with some valuable contributions to the skull region. We also employed our algorithm for chest examinations of patients aged between 1 and 5 years old. Therefore,

Medical Imaging 2014: Physics of Medical Imaging, edited by Bruce R. Whiting, Christoph Hoeschen, Despina Kontos, Proc. of SPIE Vol. 9033, 90334T · C 2014 SPIE · CCC code: 1605-7422/14/\$18 · doi: 10.1117/12.2043310 
the main purpose of this study was to quantify biological tissue in pediatric computed tomography examinations with the aim of developing and constructing patient equivalent phantoms (PEP) for pediatric patients (1-5 years old), specific to skull and chest, separately. Those phantoms shall be used in future optimization process to computed radiography systems and digital radiography systems.

\section{METHODS}

The following items describe the fundamental steps for the construction of both, chest and skull patient equivalent phantoms. The first step was to quantify biological tissues through retrospective CT examinations and to convert those biological tissues into simulator materials such as PMMA and aluminum. The second step was the construction of the pediatric homogeneous phantoms with the PMMA and aluminum plates. The examinations used in this study were all retrospective CT scans of patients aged between 1 and 5 years old, obtained in four different hospitals in the period of 2011 to 2012.

\subsection{Quantification of biological tissues by computer algorithm}

We employed the same methodology described by Pina et al (2012) for quantifying biological tissues in the chest of the newborn patients (age $<1$ year) [9]. Our methodology was designed to quantify both examinations of chest and skull, and also adapted for patients aged between 1 and 5 years old. We developed a computational algorithm in Matlab® software whose main steps are as follows:

- Read the DICOM images and the attributes present in the DICOM header;

- Align all CT slices with the vertical axis of the anatomical region;

- Estimate the anterior-posterior and lateral diameter of each CT slice;

- Generate the patient examination histogram (number of voxels x CT number);

- Make a Gaussian fitting of each tissue type in the patient examination histogram (bone, soft, fat, and lung);

- Quantify the number of voxels under each Gaussian curve;

- Determine the mean thicknesses for the biological tissues;

- Convert the mean thicknesses of the biological tissues into the corresponding thicknesses of the simulator material plates (aluminum, PMMA, and air) [10-11].

\subsection{Phantom Construction}

The results of the quantifications provided elements to construct homogeneous phantoms to simulate the infant chest and skull in the posterior-anterior or anterior-posterior (PA/AP) view. The architecture of both patient equivalent phantoms is similar to the ANSI phantom presented in AAPM Report no.31 (1990) [7], except for the thicknesses of plates, that were modified. The pediatric homogeneous phantoms were composed by PMMA and aluminum (type 1100). PMMA presents similar characteristics of soft tissue for radiation attenuation in the radiodiagnostic energy range, while aluminum represents those same characteristics for bone tissues [11].

\section{RESULTS AND DISCUSSION}

\subsection{Quantification of biological tissues}

We retrospectively analyzed $148 \mathrm{CT}$ examinations of patients aged between 1 and 5 years old, in which 56 were skull and 92 were chest examinations. After classifying the biological tissue, the computational algorithm generated the patient examination histogram. Figure 1 illustrates a chest examination histogram and Figure 2 a skull histogram. We can see in Figures 1 and 2, the distribution curves for tissue classification obtained from the Gaussian fit. The relative frequency is shown in log scale in the left axis and the Gaussian fit Values are also seen in the right axis. 


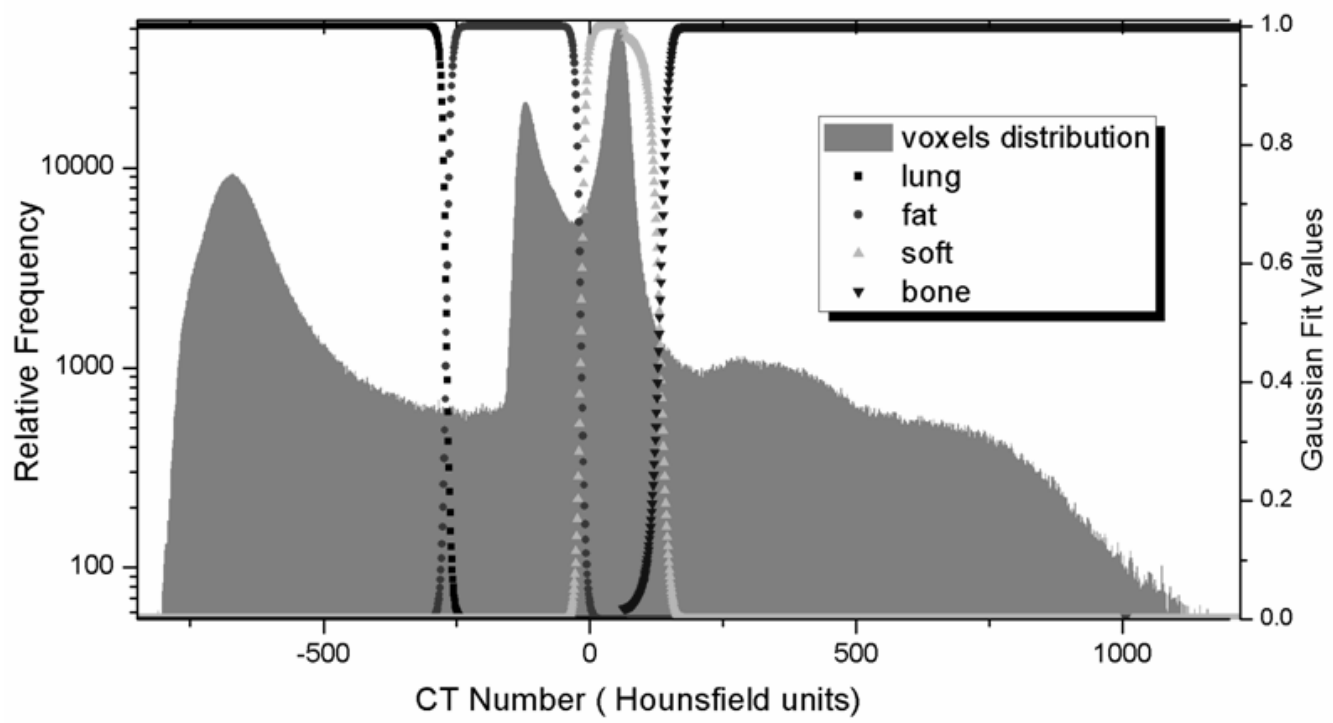

Figure 1. Chest examination histogram representing the voxel distribution in CT number and the distribution curves obtained from the Gaussian fit for each type of tissue.

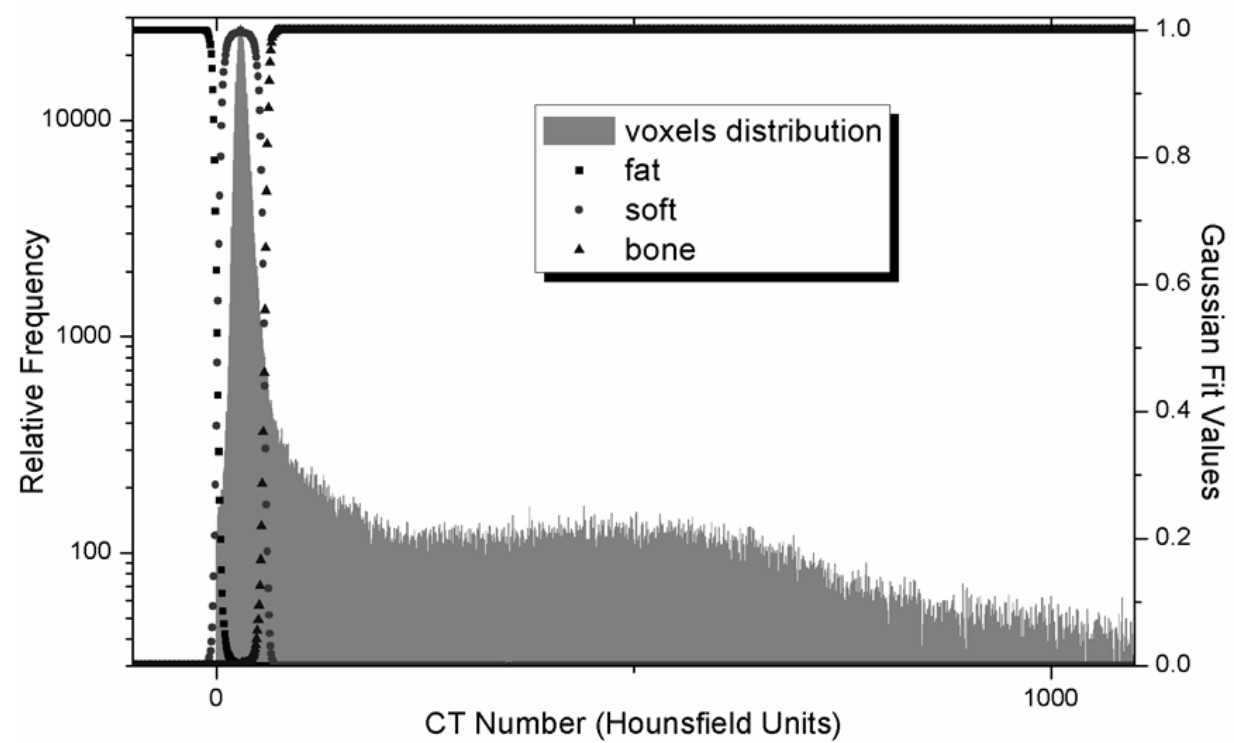

Figure 2. Skull examination histogram representing the voxel distribution in CT number and the distribution curves obtained from the Gaussian fit for each type of tissue.

When analyzing the obtained histograms, we observe the existence of specific regions with a large number of voxels that may be associated to specific biological tissues. In the chest histogram there are two pronounced and narrow peaks, near the CT-number origin. Those peaks are associated to fat and soft tissues, respectively. There is a peak whose average CT number is -700 , which corresponds to the lung tissue. The bone tissue can be seen as a much broader peak whose average CT number is 400 .

The distribution curves are also represented in the histogram. Those distribution curves were obtained by the Gaussian fit values of each tissue and its corresponding CT number, which is also the centroid value corresponded to the peaks in the histogram. There are four distributions curves dividing the histogram in four different regions, each one corresponding to a specific biological tissue. The first distribution represents the lung tissue and the second the fat tissue. The third and the 
fourth one represents soft and bone tissue, respectively. All these curves distributions were obtained by the computational algorithm. The area under peaks in each curve distribution represents the total voxel number associated to each biological tissue amount.

Regarding the skull histogram, there are some peaks that also appear in the chest histogram. There is a pronounced and narrow peak near the CT-number origin that represents fat and soft tissues. It was not necessary to differentiate these two tissues since both are converted to PMMA in the construction of the PEP. In the skull histogram there is another much broader peak whose average CT number is 600 , which corresponds to bone tissue.

After analysis of all chest and skull examinations, the amount of biological tissues classified and quantified formed a database. The content of this database is presented in Tables 1 and 2. Each table contains the following information: average thickness of biological tissues (Ti), thicknesses of simulator materials (PMMA and aluminum) and their relative uncertainty which is defined as the standard deviation of the obtained examination thickness divided by the mean value. Table 1 refers to the results of biological tissues quantification for 92 chest examinations and Table 2 to the results for the 56 skull examinations.

Table 1: Results of the chest examinations.

\begin{tabular}{|c|c|c|c|c|}
\hline Tissue & Ti $(\mathrm{mm})$ & $\begin{array}{c}\text { Simulator } \\
\text { Material }\end{array}$ & TS (mm) & $\begin{array}{c}\text { Relative } \\
\text { uncertainty (\%) }\end{array}$ \\
\hline Lung & 28.6 & Air & 28.6 & 19.4 \\
\hline Total soft & 57.2 & PMMA & 73.0 & 13.0 \\
\hline Bone & 10.2 & Aluminum & 2.3 & 20.0 \\
\hline
\end{tabular}

Table 2: Results of the skull examinations.

\begin{tabular}{|c|c|c|c|c|}
\hline Tissue & Ti $(\mathrm{mm})$ & $\begin{array}{c}\text { Simulator } \\
\text { Material }\end{array}$ & $\begin{array}{c}\text { TS } \\
(\mathrm{mm})\end{array}$ & $\begin{array}{c}\text { Relative } \\
\text { uncertainty (\%) }\end{array}$ \\
\hline Total soft & 97.5 & PMMA & 119.8 & 3.8 \\
\hline Bone & 15.1 & Aluminum & 2.7 & 9.5 \\
\hline
\end{tabular}

The results presented in Table 1 and 2 shows a significant relative uncertainty, which was due to sample variations. There are many factors that can be related to such differences. The first and most important is the age difference between patients, but there are other factors such as different ethnicities, different nutritional conditions and genetic factors.

\subsection{Phantom Construction}

The results presented in Table 1 and Table 2 were applied to the construction of the homogeneous phantoms of chest and skull respectively. The chest homogeneous phantom was constructed as follows. The first PMMA pair (at the top) sandwiched an aluminum plate. The second PMMA pair (at the bottom) contained a slightly wider aluminum slab inside. The second aluminum plate was designed to replicate the greater amount of bone tissue, which results from the presence of the vertebral column. Between the upper and lower pair of plates we inserted spacers representing the lung tissue as an air gap.

The skull homogeneous phantom was constructed with five PMMA plates of same thickness. The aluminum plates were inserted between the first and second and between the fourth and fifth PMMA plates, and they also had the same thickness. All the plates used measured $15.0 \times 15.0 \mathrm{~cm}$ for both skull and chest radiography examinations being usually performed in $18 \times 24 \mathrm{~cm}^{2}$ imaging detectors. Figure 2 illustrates the composition of both phantoms. 
(a)

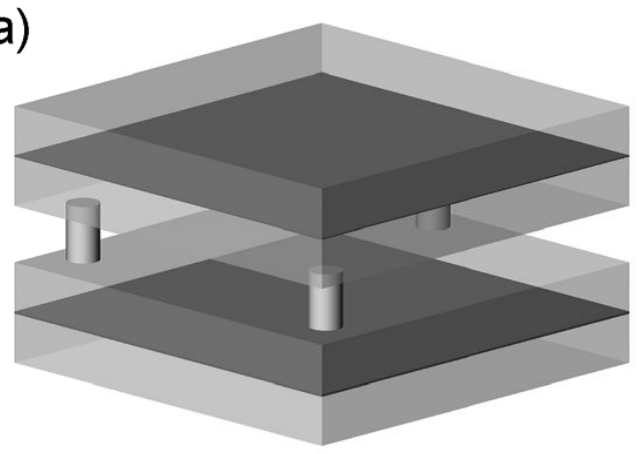

(b)

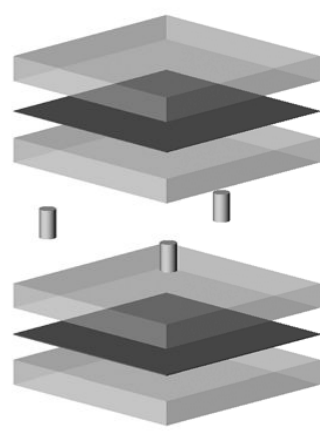

Figure 3. Illustrative view of the pediatric chest phantom. (a) Assembled set of plates. (b) Exploded view.

\section{(a)}

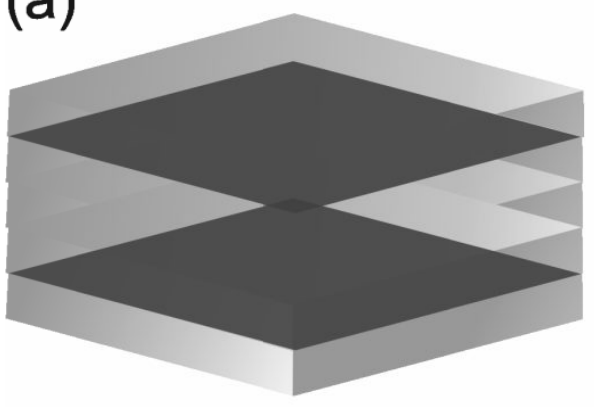

(b)

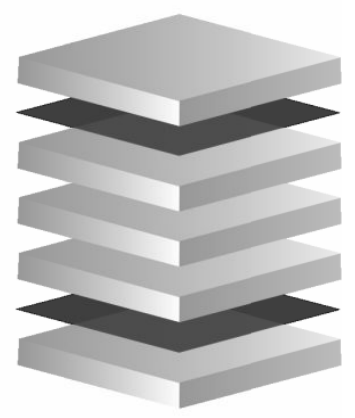

Figure 4. Illustrative view of the pediatric skull phantom. (a) Assembled set of plates. (b) Exploded view.

The pediatric homogeneous phantoms will be used in future optimization process of image quality and dose control in computed radiography and digital radiography systems.

\section{CONCLUSIONS}

It is very practical for a institution with restricted resources to have a homogeneous phantom. These phantoms are made of readily available materials such as PMMA and aluminum and may be composed of different plate thicknesses. This allows the construction of similar devices for other body regions (abdomen, lumbar spine, extremity). Although our quantification results are specific for infants between 1 and 5 years, the computational algorithm can also be easily adapted to other patient ages. In near future, we intend to evaluate the quality of radiographic image through physical variables such as noise power spectrum (NPS) and signal to noise ratio (SNR). We also intend to evaluate the dose levels received in pediatric examinations to find radiological charts to be employed in the diagnostic imaging routine. The chest and skull pediatric phantoms developed in this study are the most practical and inexpensive way to lead optimization processes in radiographic systems. This report is the first in the literature to our knowledge to describe the development of such phantoms in this specific age for pediatric patients. From this study, we intend to improve the quality of diagnosis of pediatric population and to reduce the cost of diagnosis in our institution. 


\section{REFERENCES}

[1] T. Wardlaw, P. Salama, E. W. Johansson et al., "Pneumonia: the leading killer of children," Lancet, 368(9541), 1048-50 (2006).

[2] K. S. Quayle, D. M. Jaffe, N. Kuppermann et al., "Diagnostic testing for acute head injury in children: when are head computed tomography and skull radiographs indicated?," Pediatrics, 99(5), E11 (1997).

[3] E. J. Hall, and A. J. Giaccia, [Radiobiology for the Radiologist] Lippincott Williams \& Wilkins, Philadelphia(2011).

[4] M. T. Raissaki, "Pediatric Radiation Protection," Eur Radiol Syllabus, 14, 74-83 (2004).

[5] B. J. Conway, J. E. Duff, T. R. Fewell et al., "A patient-equivalent attenuation phantom for estimating patient exposures from automatic exposure controlled x-ray examinations of the abdomen and lumbo-sacral spine," Med Phys, 17(3), 448-53 (1990).

[6] B. J. Conway, O. H. Suleiman, F. G. Rueter et al., "Patient Equivalent Attenuation Phantoms," Radiation Protection Dosimetry, 43(1-4), 123-125 (1992).

[7] R. Y. L. Chu, J. Fisher, B. R. Archer et al., [AAPM Report NO. 31 Standardized Methods for Measuring Diagnostic X-Ray Exposures] American Association of Physicists in Medicine, New York(1990).

[8] J. Vassileva, “A phantom for dose-image quality optimization in chest radiography," Br J Radiol, 75(898), 837-42 (2002).

[9] D. R. Pina, R. T. Souza, S. B. Duarte et al., "Analysis of biological tissues in infant chest for the development of an equivalent radiographic phantom," Med Phys, 39(3), 1357-60 (2012).

[10] R. J. Jennings, "A method for comparing beam-hardening filter materials for diagnostic radiology," Med Phys, 15(4), 588-99 (1988).

[11] D. R. White, "Tissue substitutes in experimental radiation physics," Med Phys, 5(6), 467-79 (1978). 